\title{
Lateralization of the Human Mirror Neuron System
}

\author{
Lisa Aziz-Zadeh, ${ }^{1,2}$ Lisa Koski, ${ }^{1,5}$ Eran Zaidel, ${ }^{2,4}$ John Mazziotta, ${ }^{1,4,5,6,7}$ and Marco Iacoboni ${ }^{1,3,4}$ \\ ${ }^{1}$ Ahmanson Lovelace Brain Mapping Center, Neuropsychiatric Institute, ${ }^{2}$ Department of Psychology, ${ }^{3}$ Department of Psychiatry and Biobehavioral \\ Sciences, ${ }^{4}$ Brain Research Institute, David Geffen School of Medicine, and Departments of ${ }^{5}$ Neurology, ${ }^{6}$ Pharmacology, and ${ }^{7}$ Radiological Sciences, \\ University of California, Los Angeles, Los Angeles, California 90095
}

\begin{abstract}
A cortical network consisting of the inferior frontal, rostral inferior parietal, and posterior superior temporal cortices has been implicated in representing actions in the primate brain and is critical to imitation in humans. This neural circuitry may be an evolutionary precursor of neural systems associated with language. However, language is predominantly lateralized to the left hemisphere, whereas the degree of lateralization of the imitation circuitry in humans is unclear. We conducted a functional magnetic resonance imaging study of imitation of finger movements with lateralized stimuli and responses. During imitation, activity in the inferior frontal and rostral inferior parietal cortex, although fairly bilateral, was stronger in the hemisphere ipsilateral to the visual stimulus and response hand. This ipsilateral pattern is at variance with the typical contralateral activity of primary visual and motor areas. Reliably increased signal in the right superior temporal sulcus (STS) was observed for both left-sided and right-sided imitation tasks, although subthreshold activity was also observed in the left STS. Overall, the data indicate that visual and motor components of the human mirror system are not left-lateralized. The left hemisphere superiority for language, then, must be have been favored by other types of language precursors, perhaps auditory or multimodal action representations.
\end{abstract}

Key words: motor; sensorimotor; premotor; language; hemisphere; lateralization

\section{Introduction}

Several studies indicate a shared representation for the execution of actions and for their observation. This finding was first observed in monkeys, where a group of neurons in the F5 region, called mirror neurons, responded to both actions made by the monkey herself and to observing the same action made by the experimenter (di Pellegrino et al., 1992; Gallese et al., 1996). Brain-imaging studies have expanded the findings to humans (Fadiga et al., 1995; Grafton et al., 1996; Rizzolatti et al., 1996b; Grezes et al., 1998, 2003; Binkofski et al., 1999; Iacoboni et al., 1999, 2005; Nishitani and Hari, 2000; Buccino et al., 2001; AzizZadeh et al., 2002; Johnson-Frey et al., 2003; Koski et al., 2003; Molnar-Szakacs et al., 2005). These studies indicate that Brodmann area 44 (BA44), which is located in the pars opercularis of the inferior frontal gyrus (probabilistic atlas) (Mazziotta et al., 2001) and is considered the human homolog of monkey area F5 (Geyer et al., 2000), may be part of the human mirror neuron system (MNS). The data further indicate that the superior temporal sulcus and the inferior parietal lobule, together with the pars opercularis, may serve as a core neural network for action

Received March 10, 2005; revised Jan. 17, 2006; accepted Jan. 18, 2006.

This work was supported by Brain Mapping Medical Research Organization, Brain Mapping Support Foundation, Pierson-Lovelace Foundation, The Ahmanson Foundation, Tamkin Foundation, Jennifer Jones-Simon Foundation, Capital Group Companies Charitable Foundation, Robson Family, Northstar Fund, and grants from the Nationa Center for Research Resources (RR12169, RR13642, and RR08655) and the National Institutes of Health (MH63680 and NS20187). We thank Stephen Wilson, Istvan Molnar-Szakacs, Eric Mooshagian, Stephen Kang, and Jonas Kaplan for their assistance with this study.

Correspondence should be addressed to Lisa Aziz-Zadeh, Ahmanson Lovelace Brain Mapping Center, CA, 660 Charles E. Young Drive South, Los Angeles, CA 90095-7085. E-mail: Isa@ucla.edu. DOI:10.1523/JNEUROSCI.2921-05.2006

Copyright $\odot 2006$ Society for Neuroscience $\quad$ 0270-6474/06/262964-07\$15.00/0 understanding (Rizzolatti et al., 2000; Carr et al., 2003; Iacoboni, 2005).

Several investigators have speculated about the ties between MNS and language evolution (Rizzolatti and Arbib, 1998; Corballis, 2002; Iacoboni, 2005). Some of this speculation is based on the anatomical location of the pars opercularis of the inferior frontal gyrus: it is a component of Broca's area, a predominant speech area in the human brain. Some have claimed that the human MNS is specialized to the left hemisphere (Corballis, 2002), the dominant hemisphere for language (Zaidel, 1978). However, this claim has not been properly tested. In the monkey, mirror neurons have been found in the F5 region of both hemispheres (Gallese et al., 1996). In humans, functional imaging studies used to examine the system have not properly controlled for laterality. For example, where a motor response is required of a participant, most studies use the right hand. Furthermore, observation of actions is often limited to watching one hand perform an action. Thus, greater activity reported in the left hemisphere MNS areas may simply reflect the laterality of either the responding hand or the observed hand.

To investigate more systematically the lateralization of the human MNS, we performed a functional magnetic resonance imaging (fMRI) study where participants were asked to observe, execute, or imitate a finger action toward a target. Because we were interested in MNS lateralization as it relates to language, we focused our analysis on lateralized patterns of activity in the pars opercularis. Stimuli were flashed to each visual hemifield and the response hand was ipsilateral to the hemifield of presentation, a method widely used to investigate behavioral differences between the two hemispheres. To our knowledge, this is the first fMRI 
Observation
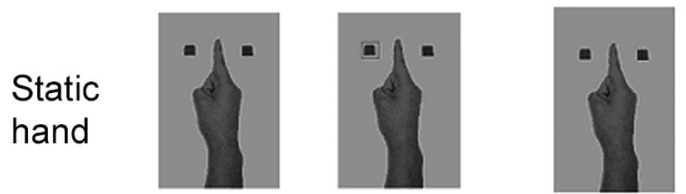

Execution
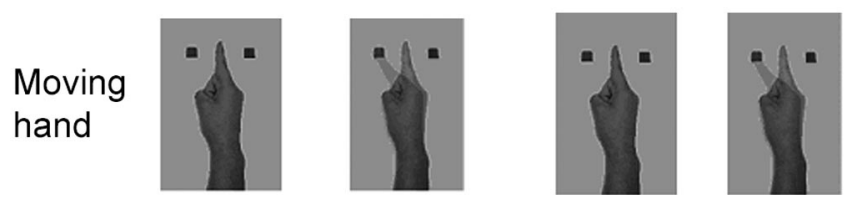

Figure 1. Stimuli used in fMRI study of action observation, execution, and imitation. Stimuli consisted of two frames of right or left hands, consecutively flashed for a total of $150 \mathrm{~ms}$ to the right or left visual field. Participants participated in four task conditions: observation of a static hand, observation of a moving hand, execution to a static hand, or imitation. The right-hand stimuli shown here all appeared to the right of a fixation cross. Left-hand stimuli appeared to the left of a fixation cross. Both left- and right-hand stimuli were presented in color.

study that controls for laterality while investigating action observation, execution, and imitation.

\section{Materials and Methods \\ Participants}

Twelve right-handed healthy volunteers (six men, six women; mean age, 29; range, 18-45) screened by questionnaire to have no history of brain damage, participated in the study. Participants gave informed consent, according to the requirements of the Institutional Review Board of the University of California, Los Angeles. Handedness was assessed by a questionnaire adapted from the Edinburgh Handedness Inventory (Oldfield, 1971). A minimum handedness score of 12 out of 15 on this inventory was needed to participate in the study. All participants reported to have normal or corrected-to-normal vision. Before scanning, participants completed a screening questionnaire to rule out medication use, a history of neurological or psychiatric disorders, substance abuse, and other medical conditions. Images were acquired using a General Electric 3.0T MRI scanner with an Advanced Magnetic Resonance Imaging upgrade for echo planar imaging (EPI).

\section{Stimuli}

Stimuli were presented in counterbalanced blocks, beginning with a rest block consisting of a fixation cross at the center of the screen. Stimuli were still images of a left or right hand on its side in an egocentric perspective with the index finger pointing forward (Fig. 1). Because data indicate that MNS activity is stronger for goal-oriented actions (Koski et al., 2002), our stimuli included a target for the hand action. Thus, a red button was present on either side of the index finger. In one-half of the blocks, the index finger moved toward either the left or right button. In the other half, the finger was still and a black box appeared around one of the buttons. Stimuli appeared for $150 \mathrm{~ms}$ in either the left visual field (LVF) or the right visual field (RVF) while participants focused on a fixation cross. Right hand stimuli were always presented to the RVF, and left hand stimuli to the LVF. Stimuli were presented in blocks, interleaved with rest blocks consisting of a center fixation cross. The order of the task blocks was counterbalanced.

\section{Task}

Participants were asked to observe, imitate, or execute actions in different blocks while their eyes remained focused on the fixation cross. Specifically, there were four conditions, two involving only observation, and two involving making a movement. In one observation condition, participants observed a goal-oriented action made by an index finger (action observation). In the other observation condition, they observed a still image of a hand (static hand observation). In one movement condition, participants imitated the action made by an index finger (imitation condition). In the other movement condition, the same action was indicated by an abstract cue (execution condition). In both movement conditions, the participant always used the right hand to respond when the righthand stimulus was presented to the RVF and the left hand to respond when the left-hand stimulus was presented to the LVF.

\section{$f M R I$}

Four scans of 5 min $56 \mathrm{~s}$ were performed with each participant. Each scan consisted of eight task periods (LVF observation of moving left hands, LVF observation of static left hands, RVF observation of moving right hands, RVF observation of static right hands, execution during LVF presentation of static left hands, execution during RVF presentation of static right hands, imitation of LVF presented left hands, imitation of RVF presented right hands) of $20 \mathrm{~s}$ alternating with nine rest periods of $20 \mathrm{~s}$. To rule out any brain abnormalities and allow prescription of the slices to be obtained in the remaining sequences, we performed a two dimensional spin echo sequence with repetition time $(\mathrm{TR})=4000 \mathrm{~ms}$, echo time $(\mathrm{TE})=40 \mathrm{~ms}, 256 \times 256$ voxel matrix, $4 \mathrm{~mm}$ thickness, and $1 \mathrm{~mm}$ spacing. Functional data were acquired using an echo planar T2weighted gradient echo sequence $(\mathrm{TR}=4000 \mathrm{~ms}$, TE $=25 \mathrm{~ms}$, flip angle $=90^{\circ}, 64 \times 64$ voxel matrix, 26 slices, $4 \mathrm{~mm}$ thickness, $1 \mathrm{~mm}$ spacing). For anatomical normalization purposes, anatomical data were acquired with a coplanar high-resolution T2-weighted echo planar imaging volume with $\mathrm{TR}=4000 \mathrm{~ms}, \mathrm{TE}=54 \mathrm{~ms}$, flip angle $=90^{\circ}, 128 \times$ 128 voxel matrix, 26 axial slices, $3.125 \mathrm{~mm}$ in-plane resolution, $4 \mathrm{~mm}$ thickness, and $1 \mathrm{~mm}$ spacing.

\section{Image processing}

Functional images were aligned to the coplanar high-resolution EPI volume within each participant using a rigid-body linear registration algorithm (Woods et al., 1998a). Image spatial normalization was performed with fifth-order polynomial nonlinear warping (Woods et al., 1998b) of each participant's images into a Talairach-compatible brain magnetic resonance atlas (Woods et al., 1999). Data were smoothed using an inplane Gaussian filter to produce a final image resolution of $8.7 \mathrm{~mm}$ by 8.7 $\mathrm{mm}$ by $8.6 \mathrm{~mm}$.

Statistical analysis was performed using an ANOVA (Woods et al., 1996). There is now a considerable body of evidence implicating the pars opercularis of the inferior frontal gyrus in action simulation, observation, execution, and imitation (Gallese et al., 1996; Grafton et al., 1996; Rizzolatti et al., 1996a; Decety et al., 1997, 2002; Krams et al., 1998; Grezes et al., 1999; Iacoboni et al., 1999, 2005; Binkofski et al., 2000; Nishitani and Hari, 2000; Buccino et al., 2001; Koski et al., 2002; Molnar-Szakacs et al., 2004). This region was characterized cytoarchitectonically and named by Brodmann as area 44 (Brodmann, 1908). It is anatomically delimited rostrally by the vertical ramus of the Sylvian fissure, dorsally by the inferior frontal sulcus, caudally by the inferior portion of the precentral sulcus, and ventrally by the Sylvian fissure. The recent analysis and probabilistic map constructed by Tomaiuolo et al. (1999) found the average volume of the pars opercularis to be $\sim 4 \mathrm{ml}$. Therefore, the statistical threshold for the pars opercularis, our primary region of interest, was set at $p=0.05$, corrected for multiple comparisons across the equivalent volume in resolution elements in our images (Worsley, 1996). The statistical threshold for brain areas outside this region of interest was set at $p=0.001$, uncorrected for multiple spatial comparisons. To circumvent the problem of blood oxygen level-dependent (BOLD) signal autocorrelation, we used as the dependent variable the sum of the signal intensity at each voxel throughout the experimental blocks (Iacoboni et al., 1999, 2001; Koski et al., 2002; Carr et al., 2003; Koski et al., 2003).

Task-related activity for all tasks minus rest. Two separate maps were generated to identify those brain regions that show task-related activity over all conditions (action execution, observation of moving hands, observation of static hands, and imitation) as compared with rest. The first map considered LVF presentations only, whereas the second map considered RVF presentations only. Each map was created from a contrast of the main effect of task minus rest using an ANOVA model with the following factors: participant $(n=12)$, run $(n=4)$, phase (task, rest), task (observation, execution), and stimulus type (moving, static). 
Task-related activity contrasting between hemispheres. Two maps were generated to identify brain regions that show task-related activity over all task conditions together (observation of static hands, observation to moving hands, execution, imitation) when stimuli are presented to the RVF as compared with the LVF or, similarly, when they are presented to the LVF as compared with the RVF. Each map was created from a contrast of the LVF (or RVF) presentation task blocks minus RVF (or LVF, respectively) presentations task blocks, using an ANOVA model with the following factors: participant $(n=12)$, run $(n=4)$, task (execution, observation), visual field (LVF, RVF) stimulus type (moving, static).

MNS-related activity. We have previously proposed an empirical way to test for MNS activity during observation, execution of action, and imitation (Iacoboni et al., 1999, 2005). Single-unit recordings in macaques show that mirror neurons respond more strongly to execution of action than its observation. During imitation, there is both observation and execution of action. Thus, one can conceptualize MNS activity as expressed by BOLD signal as progressively increasing from observation, to execution, to imitation of an action (Iacoboni et al., 1999, 2001, 2005; Koski et al., 2002, 2003). We tested this pattern with a linear contrast in frontoparietal areas known to be involved in the MNS: the pars opercularis and the posterior parietal cortex. The superior temporal sulcus (STS) is a higher order visual area, but it seems to receive efference copies of motor plans from frontoparietal mirror areas (Iacoboni et al., 2001); thus, even in STS, one might expect to find a similar pattern of activity.

MNS-related activity contrasting between hemispheres (LVF-RVF; RVF$L V F)$. We further aimed to find the areas that show an increased MNS pattern of activity when comparing laterality effects. Thus, we performed a linear contrast, comparing the areas in which BOLD signal increased progressively from observation, to execution, to imitation of an action for RVF trials more than LVF trials and vice-versa.

Mean percentage signal change in probabilistic BA44 for each condition. Because we are interested specifically in laterality for MNS areas as they relate to laterality for language, in this final analysis, we focused on BA44. We analyzed the mean percentage signal change for each task condition (imitation, execution, observation, static control) separately for each hemisphere and the stimulus/response hand in this region. We defined BA44 using probabilistic cytoarchitectonic maps (Amunts et al., 1999). These maps show, for each voxel, the percentage of subjects for whom that voxel lay in BA44. Our BA44 region of interest (ROI) included voxels in which this probability was $>20 \%$. Repeated measures ANOVA with task (imitation, execution, observation, static), cerebral hemisphere (left; right), and visual field/response hand (left; right) was also conducted to look for a main effect of hemisphere for each condition, as well as an interaction between hemisphere and response hand.

\section{Results}

\section{LVF presentations: task minus rest}

This contrast examined voxels that yielded reliable signal increases during all task periods (observation, execution, and imitation) compared with rest, when stimuli were presented to the LVF/right hemisphere. The pars opercularis in both hemispheres had reliable signal increases $(\mathrm{df}=33 ; t>2.49 ; p<0.05$, corrected for multiple spatial comparisons). Two peaks were observed in the left pars opercularis, a dorsomedial one and a ventrolateral one. A single peak was observed in the right pars opercularis. (Table 1, Fig. $2 A)$. Other areas that showed signal increases $(\mathrm{df}=$

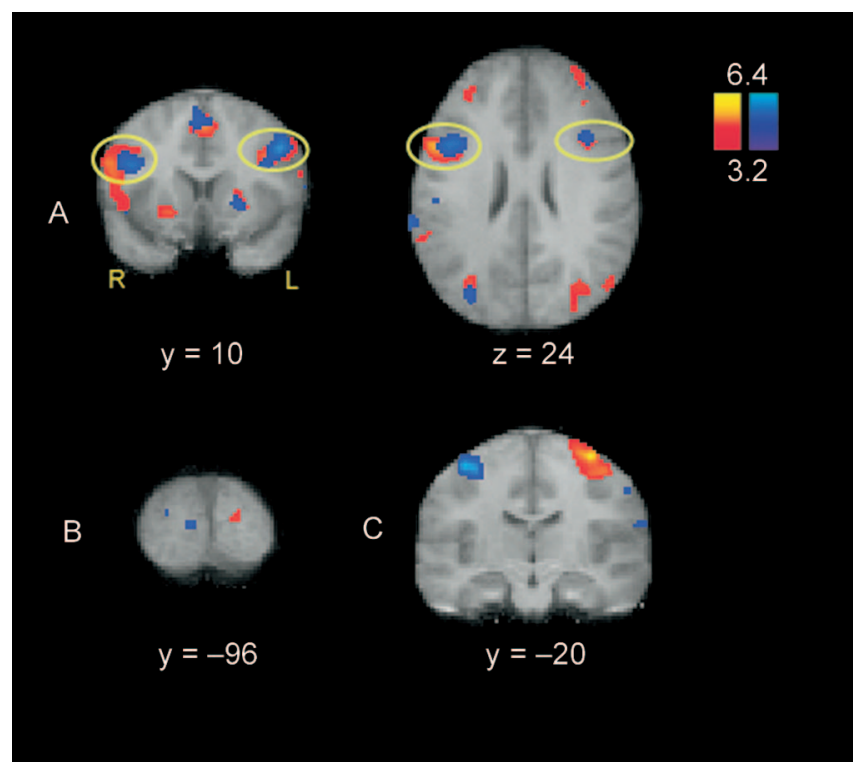

Figure 2. All activations are for the comparisons LVF-rest (blue range of voxels) and RVF-rest (red range of voxels). Activations are given as $t$ values, with the left hemisphere on the right side. Here, we show bilateral activations in pars opercularis for both comparisons $(\boldsymbol{A})$. $\boldsymbol{B}$, Lateralization of the input: activation of the contralateral visual areas. $C$, Lateralization of the output: activation of the contralateral motor areas.

$33 ; t=3.36 ; p=0.001$, uncorrected) included the right primary motor cortex, right primary visual areas, right primary somatomotor areas, right supplementary motor area (SMA), bilateral extrastriate areas, and left striatum. (Table 1, Fig. $2 B, C$ ).

\section{RVF presentations: task minus rest}

This contrast examined brain areas that yielded reliable signal increases during task periods (observation, execution, and imita- 
Table 2. Peaks of activation for brain areas active during all tasks as compared to rest for RVF trials

\begin{tabular}{|c|c|c|c|c|c|c|}
\hline \multirow[b]{2}{*}{ Hemisphere } & \multirow[b]{2}{*}{ Region } & \multirow[b]{2}{*}{ BA } & \multicolumn{3}{|c|}{ Tal-coordinates } & \multirow[b]{2}{*}{$T$} \\
\hline & & & $x$ & $y$ & $z$ & \\
\hline $\mathrm{RH}$ & Supramarginal gyrus & 40 & 62 & -45 & 24 & 3.87 \\
\hline RH & Striate & 19 & 48 & -74 & 4 & 5.80 \\
\hline RH & Pars opercularis & 44 & 54 & 7 & 26 & 5.45 \\
\hline RH & Amygdala & & 36 & 19 & 4 & 4.16 \\
\hline RH & Striate & 19 & 38 & -62 & -12 & 5.64 \\
\hline $\mathrm{RH}$ & Cerebellum & & 39 & -62 & -12 & 5.64 \\
\hline $\mathrm{RH}$ & Inferior frontal gyrus & 46 & 41 & 35 & 12 & 4.30 \\
\hline $\mathrm{RH}$ & Putamen & & 21 & 12 & -4 & 4.43 \\
\hline RH & Middle frontal gyrus & 46 & 40 & 47 & 18 & 4.82 \\
\hline RH & Middle frontal gyrus & 10 & 38 & 47 & -2 & 4.69 \\
\hline $\mathrm{RH}$ & Premotor cortex & 6 & 38 & -0.3 & 54 & 6.03 \\
\hline RH & Striate & 19 & 33 & -70 & 32 & 4.90 \\
\hline RH & Premotor cortex & 6 & 38 & 0 & 54 & 6.03 \\
\hline RH & Premotor cortex & 6 & 41 & -1 & 38 & 5.09 \\
\hline RH & Precuneus & 7 & 28 & -56 & 50 & 5.40 \\
\hline RH & Precuneus & 7 & 17 & -62 & 58 & 5.24 \\
\hline RH & Cerebellum & & 35 & -44 & -26 & 4.80 \\
\hline $\mathrm{RH}$ & Supplementary motor area & 6 & 12 & 2 & 66 & 6.69 \\
\hline LH & Supplementary motor area & 6 & 1 & 6 & 52 & 5.96 \\
\hline LH & Premotor cortex & 6 & -16 & 0 & 64 & 5.82 \\
\hline LH & Primary motor cortex & 4 & -46 & 2 & 48 & 5.69 \\
\hline LH & Premotor cortex & 6 & -30 & -5 & 60 & 6.72 \\
\hline LH & Primary motor cortex & 4 & -33 & -22 & 62 & 6.69 \\
\hline LH & Putamen & & -22 & 4.3 & 10 & 4.72 \\
\hline LH & Inferior parietal lobule & 40 & -27 & -52 & 56 & 6.14 \\
\hline LH & Striate & 19 & -24 & -84 & 38 & 6.32 \\
\hline LH & Striate & 19 & -41 & -61 & -8 & 6.51 \\
\hline LH & Pars opercularis (lateral) & 44 & -35 & 11 & 30 & 4.66 \\
\hline LH & Pars opercularis (medial) & 44 & -58 & 10 & 16 & 3.32 \\
\hline LH & Striate & $18 / 19$ & -45 & -79 & 12 & 6.72 \\
\hline
\end{tabular}

tion) compared with rest, when stimuli were presented to the $\mathrm{RVF} /$ left hemisphere. As in the previous contrast, we found bilateral signal increases in the pars opercularis $(\mathrm{df}=33 ; t=2.49 ; p=$ 0.05 , corrected for multiple spatial comparisons). A single peak in each pars opercularis was observed (Table 2, Fig. 2A). Other signal increases ( $\mathrm{df}=33 ; t=3.36 ; p=0.001$, uncorrected) were located in the left primary motor cortex, left primary visual area, bilateral SMA, premotor, posterior parietal, and extrastriate areas, and the bilateral striatum. (Table 2, Fig. $2 B, C$ ).

\section{LVF task minus RVF task}

This contrast examined brain areas that yielded a higher signal during LVF/right hemisphere presentations across task conditions (observation, execution, imitation) than during RVF/left hemisphere presentations across all task conditions. As in the previous LVF minus rest analysis, two peaks were found in the ipsilateral, left pars opercularis, a dorsomedial one and a ventrolateral one $(\mathrm{df}=33 ; t=2.49 ; p=0.05$, corrected for multiple spatial comparisons). Other differentially activated areas ( $\mathrm{df}=33 ; t=3.36$; $p=0.001$, uncorrected) included the right primary motor cortex, right visual areas, and left cerebellum.

\section{RVF task minus LVF task}

This contrast examined brain areas that yielded a higher signal during the RVF/left hemisphere presentations across task conditions (observation, execution, imitation) than during the LVF/ right hemisphere presentations across all task conditions. In keeping with the previous analysis, the ipsilateral, right pars opercularis was activated $(\mathrm{df}=33 ; t=2.49 ; p=0.05$, corrected for multiple spatial comparisons). Other activated areas ( $\mathrm{df}=33 ; t=$ 3.36; $p=0.001$, uncorrected) included the left primary motor areas, left posterior parietal, bilateral extrastriate visual areas, and right cerebellum.

\section{MNS-related activity}

Our study design included three conditions known to activate the MNS: action observation, action execution, and imitation. We looked for areas that show a pattern of activation consistent with the properties of the MNS, responding most strongly to imitation, followed by execution, then observation of actions, and minimally to observation of static images of a hand. This trend in activation pattern was explored for each VF condition as compared with rest as well as with the contralateral VF (LVF task minus RVF task, and RVF task minus LVF task conditions).

\section{Task minus rest}

As expected, the pars opercularis showed activation consistent with MNS properties. This mirror-like pattern of activity was observed not only in the left but also in the right pars opercularis. This was true both for LVF task conditions as compared with rest and for RVF task conditions as compared with rest. We also found this trend bilaterally in the posterior parietal cortex as well as in the right STS.

\section{Hemispheric contrast}

As Figure 3 shows, again the pars opercularis, posterior parietal cortex, and STS show a pattern of activity that is consistent with MNS properties. This analysis shows that when exploring for laterality, each pars opercularis is activated more strongly by the ipsilateral visual stimulus and response hand. That is, the LVFRVF contrast for MNS activity shows the left pars opercularis only and the RVF-LVF contrast shows the right pars opercularis only. This means that the pars opercularis yields reliably higher signal for ipsilateral, rather than contralateral, visual stimuli and response hands. Furthermore, like the pars opercularis, the posterior parietal cortex is more strongly activated by the ipsilateral stimulus and response hand. Other significantly active areas include the contralateral primary motor cortex and the contralateral SMA. Finally, we found the right hemisphere STS to be activated in both laterality contrasts.

Because the STS is thought to be an important region for action understanding, the right hemisphere activation observed by this contrast motivated us to investigate the laterality of this area further. Thus, we conducted a post hoc analysis in which we took the voxels in the right STS as our ROI and flipped this ROI onto the left hemisphere. Next, we extracted the mean "trends" coefficient for each subject in these two ROIs (i.e., $3 \times$ imit $+1 \times$ exec $-1 \times$ obs $-3 \times$ static) averaged over all the voxels in the ROI. This coefficient was compared in the left and right over the 12 subjects with a paired $t$ test $(\mathrm{df}=11)$, which indicated that the difference between the two ROIs was not significant ( $p=$ 0.52 ). Thus, although there was greater activation in the right STS, there must also be subthreshold activation in the left STS that also follows a mirror-like pattern of activation. 
Mean percent signal change in probabilistic BA44 for each condition

Figure 4 shows mean percent signal change as compared with rest for imitation, execution, action observation, and the control static condition for each hemisphere (left/right BA44 ROI) and each hand (left; right). The ANOVA for the signal change in probabilistic BA44 demonstrated a main effect of hemisphere, only for the execution condition $\left(F_{(1,11)}=5.76\right.$; $p=0.035)$, with the right hemisphere yielding greater signal changes than the left hemisphere. The imitation and observation conditions showed no significant differences $(p=0.46$ and $p=0.37$, respectively). Nor were there any significant main effects for visual field/response hand for the imitation, execution, or the observation condition $[p=0.47, p=0.26, p=$ 0.81 , not significant (NS), respectively].

When we tested for interactions between hemisphere and visual field/response hand, we found a highly significant interaction in the imitation condition only. The left hand shows stronger activation in the left hemisphere ROI and the right hand shows stronger activation in the right hemisphere $\operatorname{ROI}\left(F_{(1,11)}=10.94 ; p=\right.$ $0.0070)$. For execution and observation, the interactions were not significant ( $p=$ $0.91, p=0.67$, NS, respectively). No other significant effects were observed. In particular, to test whether the three experimental conditions (imitation, execution, and observation) recruited similar or different processes in probabilistic BA44, we ran an additional ANOVA, excluding the static control condition. We found no main effect of condition ( $p=0.86$; NS), and no interaction between condition and laterality (i.e., ipsilateral vs contralateral; $p=0.39$; NS).

\section{Discussion}

We found bilateral activations in the pars opercularis, independent of the visual field of presentation and the laterality of the response hand or observed hand during task trials as compared with rest trials. In both hemispheres, this area also showed an MNS-like activation pattern, with strong signal increase to imitation, less so to execution and observation, and finally even less to observation of a static hand. Thus, it seems that both the left and right pars opercularis have MNS properties and that both hemispheres contribute to the processing of action observation and imitation. This finding is further supported by an fMRI meta-analysis of 58 participants showing bilateral activation in the pars opercularis during action observation and imitation tasks (Molnar-Szakacs et al., 2005). It should be noted that when we used probabilistic BA44 to look at signal changes in this region during the tasks, this pattern did not emerge clearly (Fig. 4). This is because the large intersubject variability in cytoarchitecture results in probabilistic maps that spread into neighboring regions, making these analyses less sensitive than voxel-by-voxel analyses.

Despite the bilateral activations, when we analyzed our data by

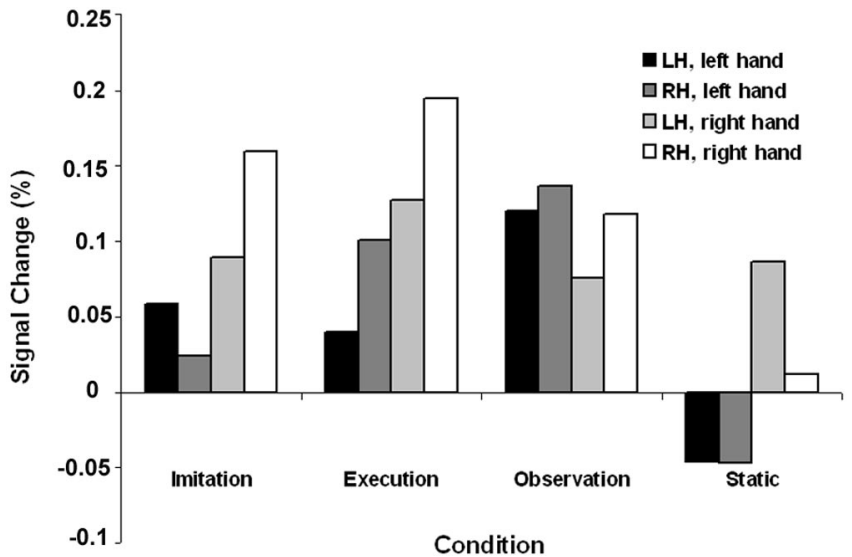

Figure 4. Mean percentage signal change as compared with rest for imitation, execution, action observation, and the control static condition for each ROI (left BA44; right BA44), and each hand (left; right). The BA44 ROI was selected using probabilistic cytoarchitectonic maps. An ANOVA indicates a significant interaction for hemisphere and hand for the imitation condition. No main effect for hemisphere was observed except for the execution condition.

comparing LVF and RVF blocks, we found greater signal increase in the ipsilateral pars opercularis. That is, our data indicate that the pars opercularis of the inferior frontal gyrus in each hemisphere is more activated for observed, executed, or imitated ipsilateral distal finger movements. The ROI analysis of signal 
changes in BA44 based on probabilistic maps of the region, however, indicated that the ipsilateral pattern is reliable only in the imitation condition.

This ipsilateral pattern of activity, albeit seemingly paradoxi$\mathrm{cal}$, is indeed consistent with findings in previous fMRI studies in ventral premotor regions involved in distal finger movements. These studies indicate that performing a precision grip is associated with greater signal in the inferior frontal gyrus ipsilateral to the responding hand (Ehrsson et al., 2000), and that the ventral premotor cortex has ipsilateral finger representations (Cramer et al., 1999; Hanakawa et al., 2005). Furthermore, both left- and right-brain damaged patients show grasping deficits with the ipsilesional hand (Hermsdorfer et al., 1999). Together, these studies indicate that there is a greater ipsilateral response in ventral premotor cortex during finger movements, and such a pattern emerges more readily during imitation of finger movements, as our data suggest.

The ipsilateral activation in pars opercularis may be attributed to the visual field, laterality of the observed hand, laterality of the response hand, or any combination of these three variables. Because the laterality of the activation is influenced by the laterality of the hand used or observed, the data indicate quite clearly that caution must be used in designing and interpreting action observation/imitation studies where laterality is of theoretical importance. The laterality of the effector, of the stimulus, and of the side of space in which the stimulus is presented may show differentially lateralized activations in the system. The perspective of the observed action (egocentric or allocentric orientations) may also influence laterality patterns and should be explored in future studies.

The inferior frontal cortex has prominent connections with the STS via the arcuate fasciculus and with the inferior parietal lobule (Catani et al., 2005). Although the STS is not considered a mirror neuron area because it is not activated for action execution, it is thought to provide a visual description of the action that is critical for the process of imitation, and therefore considered to be strongly associated with mirror neuron areas. Together, these three areas are thought to constitute a network critical for imitation (Nishitani and Hari, 2002; Carr et al., 2003; Miall, 2003; Iacoboni, 2005). We found activation in the rostral section of the inferior parietal lobule to be stronger in the hemisphere ipsilateral to the hand observed, imitated, or moved. It appears that this area, which seems to correspond to area PF in the monkey brain (Iacoboni et al., 2006), also has stronger ipsilateral than contralateral representation. Furthermore, our data indicate that the STS bilaterally shows a mirror-like pattern of activation, although the activation is stronger in the right hemisphere. However, such pattern is unlikely caused by motor activity originating from STS, and likely reflects efferent copies of motor commands from MNS areas back to STS (Iacoboni et al., 2001).

A previous study also found differing lateralized activations in the inferior parietal cortex and superior temporal gyrus based not on the effector used (only right hands were manipulated) but on whether one is imitating another person or another person is imitating them (Decety et al., 2002). It is difficult to interpret those results in relation to the current ones because the former study only manipulated the right hand, whereas our data focus on differences between manipulating either the left or right effector. However, it is possible that, although the effector observed, imitated, or moved contributes to ipsilateral activations, other task demands, such as focus on the self or other [or, as in another study, possibly deficits in mentalization (Nishitani et al., 2004)], may also have strong contributions to lateralized findings.
To conclude, these data suggest that the human frontoparietal mirror neuron system is bilaterally distributed in its activity. Aspects of left lateralization of language, then, must be accounted for by other factors. Recently, auditory mirror neurons have been discovered in the macaque brain (Kohler et al., 2002). In our lab, using transcranial magnetic stimulation, we observed a leftlateralized corticospinal facilitation to action sounds (AzizZadeh et al., 2004). A recent fMRI study has also found stronger left ventral premotor activation to action sounds (Gazzola et al., 2005). Thus, one possibility is that the progression toward left lateralization of language functions may have been facilitated by a left-lateralized auditory component of a multimodal mirror neuron system.

\section{References}

Amunts K, Schleicher A, Burgel U, Mohlberg H, Uylings HB, Zilles K (1999) Broca's region revisited: cytoarchitecture and intersubject variability. J Comp Neurol 412:319-341.

Aziz-Zadeh L, Maeda F, Zaidel E, Mazziotta J, Iacoboni M (2002) Lateralization in motor facilitation during action observation: a TMS study. Exp Brain Res 144:127-131.

Aziz-Zadeh L, Iacoboni M, Zaidel E, Wilson S, Mazziotta J (2004) Left hemisphere motor facilitation in response to manual action sounds. Eur J Neurosci 19:2609-2612.

Binkofski F, Buccino G, Stephan KM, Rizzolatti G, Seitz RJ, Freund HJ (1999) A parieto-premotor network for object manipulation: evidence from neuroimaging. Exp Brain Res 128:210-213.

Binkofski F, Amunts K, Stephan KM, Posse S, Schormann T, Freund H-J, Zilles K, Seitz RJ (2000) Broca's region subserves imagery of motion: A combined cytoarchitectonic and fMRI study. Hum Brain Mapp $11: 273-285$.

Brodmann K (1908) Beitrage zur histologischen lokalisation der grosshirnrinde. VI. Mitteilung: die cortexgliederung des menschen. J Psychol Neurol (Lzp) 10:231-246.

Buccino G, Binkofski F, Fink GR, Fadiga L, Fogassi L, Gallese V, Seitz RJ, Zilles K, Rizzolatti G, Freund HJ (2001) Action observation activates premotor and parietal areas in a somatotopic manner: an fMRI study. Eur J Neurosci 13:400-404.

Carr L, Iacoboni M, Dubeau MC, Mazziotta JC, Lenzi GL (2003) Neural mechanisms of empathy in humans: A relay from neural systems for imitation to limbic areas. Proc Natl Acad Sci USA 100:5497-5502.

Catani M, Jones DK, ffytche DH (2005) Perisylvian language networks of the human brain. Ann Neurol 57:8-16.

Corballis MC (2002) From hand to mouth: The origins of language. New Jersey: Princeton UP.

Cramer SC, Finklestein SP, Schaechter J, Bush G, Rosen BR (1999) Activation of distinct motor cortex regions during ipsilateral and contralateral finger movements. J Neurophysiol 81:383-387.

Decety J, Grezes J, Costes N, Perani D, Jeannerod M, Procyk E, Grassi F, Fazio F (1997) Brain activity during observation of actions. Influence of action content and subject's strategy. Brain 120:1763-1777.

Decety J, Chaminade T, Grezes J, Meltzoff AN (2002) A PET exploration of the neural mechanisms involved in reciprocal imitation. NeuroImage 15:265-272.

di Pellegrino G, Fadiga L, Fogassi L, Gallese V, Rizzolatti G (1992) Understanding motor events: a neurophysiological study. Exp Brain Res 91:176-180.

Ehrsson HH, Fagergren A, Jonsson T, Westling G, Johansson RS, Forssberg H (2000) Cortical activity in precision- versus power-grip tasks: an fMRI study. J Neurophysiol 83:528-536.

Fadiga L, Fogassi L, Pavesi G, Rizzolatti G (1995) Motor facilitation during action observation: a magnetic stimulation study. J Neurophysiol 73:2608-2611.

Gallese V, Fadiga L, Fogassi L, Rizzolatti G (1996) Action recognition in the premotor cortex. Brain 119:593-609.

Gazzola V, Aziz-Zadeh L, Keysers C (2005) I hear what you are doing: an fMRI study of the auditory mirror system in humans. In: Cognitive Neuroscience Society abstracts. New York: Cognitive Neuroscience Society.

Geyer S, Matelli M, Luppino G, Zilles K (2000) Functional neuroanatomy of the primate isocortical motor system. Anat Embryol (Berl) 202:443-474. 
Grafton ST, Arbib MA, Fadiga L, Rizzolatti G (1996) Localization of grasp representations in humans by positron emission tomography. 2. Observation compared with imagination. Exp Brain Res 112:103-111.

Grezes J, Costes N, Decety J (1998) Top-down effect of strategy on the perception of human biological motion: A PET investigation. Cognitive Neuropsychology 15:553-582.

Grezes J, Costes N, Decety J (1999) The effects of learning and intention on the neural network involved in the perception of meaningless actions. Brain 122:1875-1887.

Grezes J, Armony JL, Rowe J, Passingham RE (2003) Activations related to "mirror" and "canonical" neurones in the human brain: an fMRI study. NeuroImage 18:928-937.

Hanakawa T, Parikh S, Bruno MK, Hallett M (2005) Finger and face representations in the ipsilateral precentral motor areas in humans. J Neurophysiol 93:2950-2958.

Hermsdorfer J, Laimgruber K, Kerkhoff G, Mai N, Goldenberg G (1999) Effects of unilateral brain damage on grip selection, coordination, and kinematics of ipsilesional prehension. Exp Brain Res 128:41-51.

Iacoboni M (2005) Understanding others: imitation, language, empathy. In: Perspectives on imitation: imitation in animals (Hurley S, Chater N, eds). Cambridge, MA: MIT.

Iacoboni M, Woods RP, Brass M, Bekkering H, Mazziotta JC, Rizzolatti G (1999) Cortical mechanisms of human imitation. Science 286:2526-2528.

Iacoboni M, Koski LM, Brass M, Bekkering H, Woods RP, Dubeau MC, Mazziotta JC, Rizzolatti G (2001) Reafferent copies of imitated actions in the right superior temporal cortex. Proc Natl Acad Sci USA 98:13995-13999.

Iacoboni M, Molnar-Szakacs I, Gallese V, Buccino G, Mazziotta JC, Rizzolatti G (2005) Grasping the intentions of others with one's own mirror neuron system. PLoS Biol 3:1-7.

Iacoboni M, Kaplan J, Wilson S (2006) A neural architecture for imitation and intentional relations. In: Imitation and social learning in robots, humans and animals: behavioural, social and communicative dimensions (Nehaniv C, Dautenhahn K, eds). Cambridge, UK: Cambridge UP, in press.

Johnson-Frey SH, Maloof FR, Newman-Norlund R, Farrer C, Inati S, Grafton ST (2003) Actions or hand-object interactions? Human inferior frontal cortex and action observation. Neuron 39:1053-1058.

Kohler E, Keysers C, Umilta MA, Fogassi L, Gallese V, Rizzolatti G (2002) Hearing sounds, understanding actions: action representation in mirror neurons. Science 297:846-848.

Koski L, Wohlschlager A, Bekkering H, Woods RP, Dubeau MC, Mazziotta JC, Iacoboni M (2002) Modulation of motor and premotor activity during imitation of target-directed actions. Cereb Cortex 12:847-855.

Koski L, Iacoboni M, Dubeau MC, Woods RP, Mazziotta JC (2003) Modulation of cortical activity during different imitative behaviors. J Neurophysiol 89:460-471.

Krams M, Rushworth MFS, Deiber M-P, Frackowiak RSJ, Passingham RE (1998) The preparation, execution and suppression of copied movements in the human brain. Exp Brain Res 120:386-398.

Mazziotta J, Toga A, Evans A, Fox P, Lancaster J, Zilles K, Woods R, Paus T, Simpson G, Pike B, Holmes C, Collins L, Thompson P, MacDonald D, Iacoboni M, Schormann T, Amunts K, Palomero-Gallagher N, Geyer S,
Parsons L, et al. (2001) A probabilistic atlas and reference system for the human brain: International consortium for brain mapping (ICBM). Philos Trans R Soc Lond B Biol Sci 356:1293-1322.

Miall RC (2003) Connecting mirror neurons and forward models. NeuroReport 14:2135-2137.

Molnar-Szakacs I, Iacoboni M, Koski L, Mazziotta JC (2005) Functional segregation within pars opercularis of the inferior frontal gyrus: evidence from fMRI studies of imitation and action observation. Cereb Cortex 15:986-994.

Nishitani N, Hari R (2000) Temporal dynamics of cortical representation for action. Proc Natl Acad Sci USA 97:913-918.

Nishitani N, Hari R (2002) Viewing lip forms: cortical dynamics. Neuron 36:1211-1220.

Nishitani N, Avikainen S, Hari R (2004) Abnormal imitation-related cortical activation sequences in Asperger's syndrome. Ann Neurol 55:558-562.

Oldfield RC (1971) The assessment and analysis of handedness: the Edinburgh inventory. Neuropsychologia 9:97-113.

Rizzolatti G, Arbib MA (1998) Language within our grasp. Trends Neurosci 21:188-194.

Rizzolatti G, Fadiga L, Gallese V, Fogassi L (1996a) Premotor cortex and the recognition of motor actions. Brain Res Cogn Brain Res 3:131-141.

Rizzolatti G, Fadiga L, Matelli M, Bettinardi V, Paulesu E, Perani D, Fazio F (1996b) Localization of grasp representations in humans by PET: 1. Observation versus execution. Exp Brain Res 111:246-252.

Rizzolatti G, Fogassi L, Gallese V (2000) Cortical mechanisms subserving object grasping and action recognition: a new view on the cortical motor functions. In: The new cognitive neurosciences (Gazzaniga MS, ed). Cambridge: MIT.

Tomaiuolo F, MacDonald JD, Caramanos Z, Posner G, Chiavaras M, Evans AC, Petrides M (1999) Morphology, morphometry and probability mapping of the pars opercularis of the inferior frontal gyrus: an in vivo MRI analysis. Eur J Neurosci 11:3033-3046.

Woods RP, Iacoboni M, Grafton ST, Mazziotta JC (1996) Improved analysis of functional activation studies involving within-subject replications using a three-way ANOVA model. In: Quantification of brain function using PET (Myers R, Cunningham V, Bailey D, Jones T, eds), pp 353-358. San Diego: Academic.

Woods RP, Grafton ST, Holmes CJ, Cherry SR, Mazziotta JC (1998a) Automated image registration: I. General methods and intrasubject, intramodality validation. J Comput Assist Tomogr 22:139-152.

Woods RP, Grafton ST, Watson JD, Sicotte NL, Mazziotta JC (1998b) Automated image registration: II. Intersubject validation of linear and nonlinear models. J Comput Assist Tomogr 22:153-165.

Woods RP, Dapretto M, Sicotte NL, Toga AW, Mazziotta JC (1999) Creation and use of a Talairach-compatible atlas for accurate, automated, nonlinear intersubject registration, and analysis of functional imaging data. Hum Brain Mapp 8:73-79.

Worsley KJ (1996) A unified statistical approach for determining significant signals in images of cerebral activation. Hum Brain Mapp 4:58-73.

Zaidel E (1978) Concepts of cerebral dominance in the split brain. In: Cerebral correlates of conscious experience (Rougeul-Buser A, RougeulBuser P, eds), pp 263-284. Amsterdam: Elsevier. 